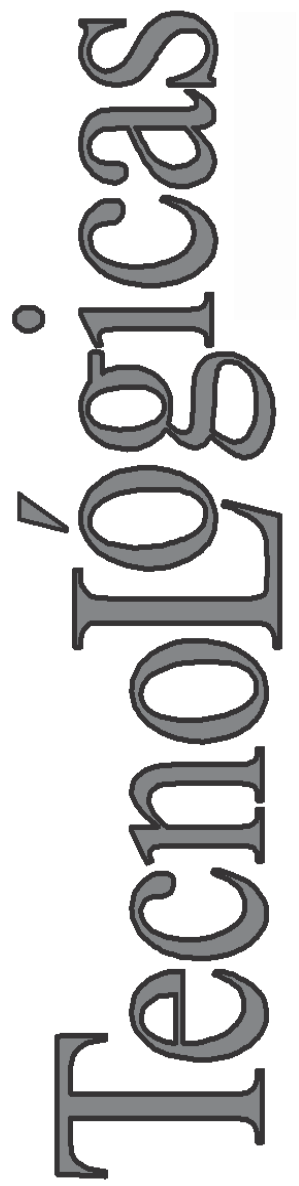

\title{
Diseño de Controlador Robusto para Horno Rotatorio
}

\section{Design Robust Controller for Rotary Kiln}

\author{
Omar D. Hernández-Arboleda ${ }^{1}$ \\ Paula A. Ortiz-Valencia ${ }^{2}$ \\ Jorge A. Herrera-Cuartas ${ }^{3}$
}

1 Cementos Argos S.A., Medellín-Colombia ohernandez@argos.com.co

2 Facultad de Ingeniería, Instituto Tecnológico Metropolitano, Medellín-Colombia paulaortiz@itm.edu.co

3 Facultad de Ingeniería, Instituto Tecnológico Metropolitano, Medellín-Colombia jorgeherrera@itm.edu.co 


\section{Resumen}

En este artículo se presenta el diseño de un controlador robusto para un horno rotativo. El controlador diseñado es una combinación de un PID fraccionario y un regulador cuadrático lineal (LQR por su sigla en inglés, Linear Quadratic Regulator), los cuales no se han usado, hasta ahora, para controlar un horno rotatorio, además se evalúan los criterios de robustez (margen de ganancia, margen de fase, robustez de ganancia, sensibilidad y rechazo de ruido de alta frecuencia) aplicados al modelo completo (controlador-planta), obteniendo óptimos resultados en un rango de frecuencia de 0.020 a $90 \mathrm{rad} / \mathrm{s}$, lo cual contribuye con la robustez del sistema.

\section{Palabras clave}

Control fraccional; control LQR; control robusto; horno rotatorio.

\section{Abstract}

This paper presents the design of a robust controller for a rotary kiln. The designed controller is a combination of a fractional PID and linear quadratic regulator (LQR), these are not used to control the kiln until now, in addition robustness criteria are evaluated (gain margin, phase margin, strength gain, rejecting high frequency noise and sensitivity) applied to the entire model (controller-plant), obtaining good results with a frequency range of 0.020 to $90 \mathrm{rad} / \mathrm{s}$, which contributes to the robustness of the system.

\section{Keywords}

Robust control; LQR control; fractional control; rotary kiln. 


\section{INTRODUCCIÓN}

Un horno rotativo (CRK) es un cilindro largo, ligeramente inclinado y giratorio que se utiliza en el proceso continuo de calcinación de pasta a temperaturas superiores a $1400^{\circ} \mathrm{C}$ para producir clinker en la industria del cemento. El CRK es el corazón de la cadena de fabricación de cemento, cuya capacidad, en general, determina la capacidad total de la planta. El proceso de calcinación es un proceso con alto consumo de energía y genera grandes cantidades de polvo y de emisiones de gases de efecto invernadero. Se estima que el horno consume más del $80 \%$ de la energía térmica que se requiere en una planta de cemento. El horno es el responsable del $5 \%$ al $7 \%$ del $\mathrm{CO}_{2}$ producido por el hombre, el cual puede alcanzar hasta un $25 \%$ para el año 2050 teniendo en cuenta la demanda actual creciente, cada vez mayor, de cemento. En consecuencia, la mejora en la eficiencia de la combustión del CRK y la reducción de emisión de $\mathrm{CO}_{2}$, son las principales preocupaciones en el contexto del desarrollo sostenible de la industria cementera tratada en el protocolo de Kyoto (Kyoto, 1997) y el Consejo Empresarial Mundial para el Desarrollo Sostenible. Para lograr este objetivo, el primer paso es el seguimiento y control del proceso (Shahriari \& Tarasiewicz, 2009).

Hasta ahora, los controles propuestos para los hornos rotatorios han centrado su atención en tomar el horno como una caja gris o negra de múltiples entradas y múltiples salidas y aplican a este modelo técnicas de control inteligente como redes neuronales (Pani, Vadlamudi, \& Mohanta, 2013; Lin, Liu, Cao, \& Huang, 2009; Lin, Liu, Song, \& Song, 2009); control difuso (Yongjian, Minghao, Xiaojie, \& Tianyou, 2007; Feng, Bin, Xiaochen, \& Peng, 2010; Holmblad \& Østergaard, 1995) o la combinación de varias de estas técnicas con otras como los PID clásicos (P. Wang \& Kwok, 1992) o las máquinas de soporte vectorial (SVM) (Li, 2010). Otros trabajos llevan a la construcción de sistemas expertos para tratar de controlar el horno (C. Wang, Wang, Yu, \& Li, 2010; S. Wang, Dong, \& Yuan, 2007). Estos controles garantizan la operación del horno en condiciones estables, pero la robustez de tales controles no ha sido evaluada por ninguno de sus autores. 
Dado lo anterior, se propone la implementación de un controlador robusto tipo PID fraccionario con ganancia proporcional LQR "Linear Quadratic Regulator", y para el cual se hace un análisis de la robustez del sistema completo (planta-controlador). El trabajo está organizado de la siguiente forma: En la sección 2 se presenta el modelo de la planta a controlar. El diseño del controlador robusto se presenta en la sección 3. En la sección 4 se muestran los criterios de robustez con los cuales se evaluó el sistema controlador-planta. Los resultados obtenidos y su discusión se presentan en la sección 5; y finalmente, las conclusiones se presentan en la sección 6.

\section{MODELO DEL SISTEMA}

Para modelar el proceso de combustión se utiliza el principio de la temperatura adiabática de llama y el factor de utilización de calor expuesto en (Marquez Martinez, 1989). La Fig. 1 muestra el esquema general propuesto para determinar el modelo estático del proceso de clinkerización. Se aprecia el calor disponible por la transformación del combustible en el quemador del proceso y las diferentes etapas en las cuales se emplea dicho calor, como la transformación de la materia prima en Clinker, la evaporación de agua y las pérdidas de calor en la coraza del horno. Este balance da como resultado el calor que sobra en el proceso y que se refleja en la temperatura de los gases de salida del horno.

La base matemática y física del modelo estático están sustentadas en las leyes de la termodinámica clásica; este modelo sirve como soporte para encontrar el modelo dinámico, pues a partir de las variables de entrada, la variable de salida del modelo estático y la variable real, se realiza la identificación para hallar el modelo de orden fraccionario dinámico, el cual determina el comportamiento fraccionario del proceso de combustión del horno. El modelo dinámico representa el comportamiento del proceso ante variaciones de algunos de sus parámetros en el dominio del tiempo o de la frecuencia. Para hallar el modelo de orden fraccionario dinámico se utilizó la teoría expuesta en (Vinagre \& Monje, 2006) para sistemas de orden conmensurado y racional. Los datos empleados 
son tomados de un calentamiento del horno, donde la dinámica del sistema queda determinada por los incrementos o decrementos de las variables de entrada (ratas de combustible, rata de pasta y rata de agua) y las variaciones que genera en la variable de salida (temperatura de los gases de salida). Con los datos obtenidos del modelo y los datos reales de la variable de salida se hace la identificación de un sistema SISO ("Single Input single Output") de orden fraccionario. La estructura del modelo completo se muestra en la Fig. 2.

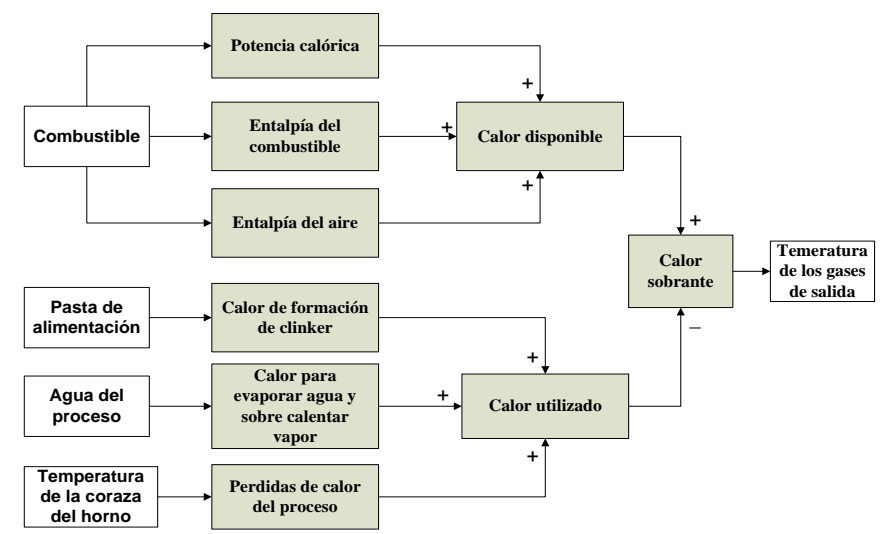

Fig. 1. Esquema general del modelo estático. Fuente: Autores

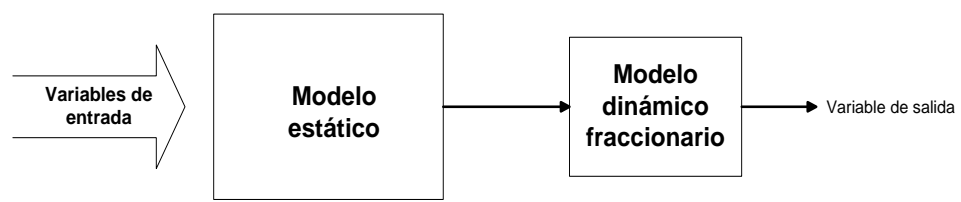

Fig. 2. Modelo completo del proceso. Fuente: Autores

\section{DISEÑO DEL CONTROL ROBUSTO}

La metodología propuesta es hallar un controlador PID de orden fraccionario utilizando la estructura SISO y aplicar este controlador a los actuadores de las tres variables consideradas criticas del proceso (carbón, pasta y agua). La estructura del controlador 
PID fraccionario con ganancia proporcional LQR se muestra en la Fig. 3.

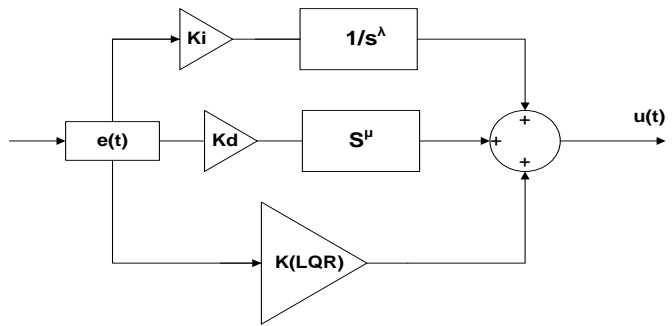

Fig. 3. Controlador PID fraccionario con ganancia LQR. Fuente: Autores

Para el cálculo de la ganancia proporcional aplicando un control robusto LQR es necesario obtener el modelo lineal del proceso, una vez linealizado el sistema, se procede a diseñar el control, donde los parámetros que se desean optimizar son la rapidez, el error y la energía. Para esto se debe seleccionar un índice de desempeño cuadrático, el cual se representa en (1).

$J=\frac{1}{2} \int_{0}^{\infty}\left(x^{T} Q x+u^{T} R u\right) d t$

Donde $Q$ es una matriz simétrica, semidefinida positiva de dimensiones, R es una matriz simétrica definida positiva de dimensiones. Para calcular la matriz de ganancias óptimas se utilizan (2) y (3).

$K=R^{-1} B P$

$P A+A^{T} P-P B R^{-1} B^{T} P+Q=0$

La expresión (4) es la ecuación de Riccati, la cual se utiliza para hallar la matriz P. Después de obtener la matriz $\mathrm{P}$ se obtiene la matriz $\mathrm{K}$ de ganancias de los controladores usando (2). Una vez encontrada la ganancia proporcional LQR se ajusta la ganancia integral y derivativa del sistema de orden fraccional, la expresión se muestra en (4). Donde $\lambda$ y $\mu$ son números reales entre cero y 
dos. Para el ajuste de los valores de $K_{i}, K_{d}, \lambda$ y $\mu$ se tiene en cuenta los siguientes criterios de robustez.

$P I^{\lambda} D^{\mu}=G_{c}(s)=K_{p(L Q R)}+K_{i} s^{-\lambda}+K_{d} s^{\mu}$

\section{CRITERIOS DE ROBUSTEZ}

\subsection{Margen de Fase y Ganancia en la Frecuencia de Corte}

El margen de fase en los sistemas de orden entero define el grado de amortiguamiento de la respuesta transitoria que tiene el sistema en el dominio del tiempo. En (5) se define el criterio del margen de fase y ganancia en la frecuencia de corte $\omega \mathrm{cg}$.

$\arg (C(j \omega c g) G(j \omega c g))=-\pi+\phi m$

$|C(j \omega c g) G(j \omega c g)|_{d B}=0 d B$

\subsection{Robustez ante Variaciones de Ganancia de la Planta}

Esta condición está representada en (6) y muestra el cambio de la fase de lazo abierto $\mathrm{F}(\mathrm{s})=\mathrm{C}(\mathrm{s}) \mathrm{G}(\mathrm{s})$, dicho cambio debe ser igual a cero en un rango de frecuencias alrededor de la frecuencia de corte $\omega c \mathrm{c}$.

$\left.\frac{d[\arg (F(s))]}{d \omega}\right|_{\omega=\omega_{c g}}=0$

Se observa en (7) que la fase de lazo abierto $\mathrm{F}(\mathrm{s})$ se fuerza a ser plana alrededor de la frecuencia de corte $\omega c \mathrm{c}$, lo cual implica que en ese rango de frecuencia la fase sea casi constante. Esto significa que ante cambios de ganancia de la planta, el sobrepaso de la respuesta transitoria es casi constante, propiedad conocida como iso-amortiguamiento. El rango de ganancias donde se cumple esta condición no puede ser fijado a voluntad por el diseñador del control; este rango depende de las características de frecuencia de la planta y del controlador resultante. 


\subsection{Rechazo a Ruido de Alta Frecuencia}

Para esta condición se utiliza la función complementaria de la función de sensibilidad que se muestra en (7). Donde A es la atenuación al ruido de alta frecuencia $\omega t$.

$\left|T(j \omega)=\frac{C(j \omega) G(j \omega)}{1+C(j \omega) G(j \omega)}\right|_{d B} \leq A d B$
$\forall \omega \geq \omega_{t} \mathrm{rad} / \mathrm{s} \Rightarrow|T(j \omega)|_{d B}=A d B$

\subsection{Rechazo a Perturbaciones de Salida}

Esta condición utiliza la función de sensibilidad que se muestra en (8).

$\left|S(j \omega)=\frac{1}{1+C(j \omega) G(j \omega)}\right|_{d B} \leq B d B$
$\forall \omega \leq \omega_{s} \mathrm{rad} / s \Rightarrow|S(j \omega)|_{d B}=B d B$

Donde B es el valor de sensibilidad para frecuencias $\omega$ menores a $\omega s$.

\section{RESULTADOS Y ANÁLISIS}

\subsection{Controlador}

El primer paso fue obtener el modelo lineal del proceso para las variables de estado seleccionadas (carbón, pasta y agua) utilizando el modelo del proceso validado previamente. Luego se establecieron las matrices Q y R para los pesos asignados a las señales de control y los esfuerzos de los controladores. Se asignó el mayor peso al carbón porque es la variable de mayor impacto en el proceso por ser el aportante de la energía y por ende de gran impacto financiero y ambiental. Finalmente se obtiene la matriz K.

Los valores de los parámetros para los controladores PID fraccionario y con ganancia proporcional LQR se muestran en la Tabla 1. Las ganancias negativas se deben a las acciones inversas de los 
controladores con respecto a la señal de salida, y es el comportamiento lógico del proceso, si se aumenta la pasta o el agua de enfriamiento se disminuye la temperatura de los gases de salida y viceversa. Mientras que el carbón tiene una acción directa porque un aumento en la dosificación implica un aumento de temperatura en los gases de salida y viceversa. El comportamiento del sistema se observa en la Fig. 4 y en la Fig. 5 se observa el comportamiento del controlador de carbón y se aprecia el efecto directo del mismo.

Tabla 1. Parámetros controladores PID fraccionarios. Fuente: Autores

\begin{tabular}{cccc}
\multicolumn{4}{c}{ Controladores } \\
\hline Parámetro & Carbón & Pasta & Agua \\
$\mathrm{Kp}$ & 0.136800 & -0.240200 & -0.379400 \\
$\mathrm{Ki}$ & 0.090333 & 0.090333 & 0.090333 \\
$\mathrm{Kd}$ & 0.010000 & 0.010000 & 0.010000 \\
$\mathrm{\Lambda}$ & 1.248800 & 1.248800 & 1.248800 \\
$\mu$ & 0.178770 & 0.178770 & 0.178770 \\
\hline
\end{tabular}

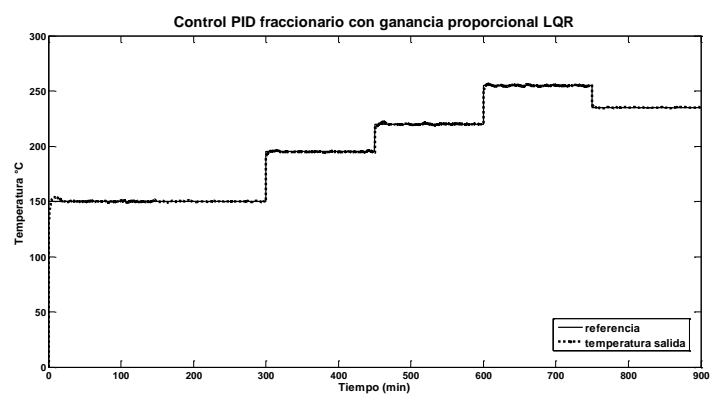

Fig. 4. Efecto de controladores PID fraccionario con ganancias LQR. Fuente: Autores

\subsection{Verificación de los Criterios de Robustez}

La Fig. 6 muestra el margen de fase y de ganancia del sistema a lazo abierto, en él se observa que el margen de fase cerca de $0 \mathrm{~dB}$ es aproximadamente $99.3^{\circ}$ y la frecuencia es $15.48 \mathrm{rad} / \mathrm{s}$. Se observa que la fase es plana entre $0.00143 \mathrm{rad} / \mathrm{s}$ y $0.01458 \mathrm{rad} / \mathrm{s}$, donde el margen de fase es $67.8^{\circ}$ aproximadamente, en este rango la ganancia varía entre $45.55 \mathrm{~dB}$ y $70.61 \mathrm{~dB}$, lo cual permite un margen de ganancia de robustez de $25.6 \mathrm{~dB}$. Con esto se verifica que el 
sistema es robusto en el rango de frecuencias y ganancias expuesto anteriormente.

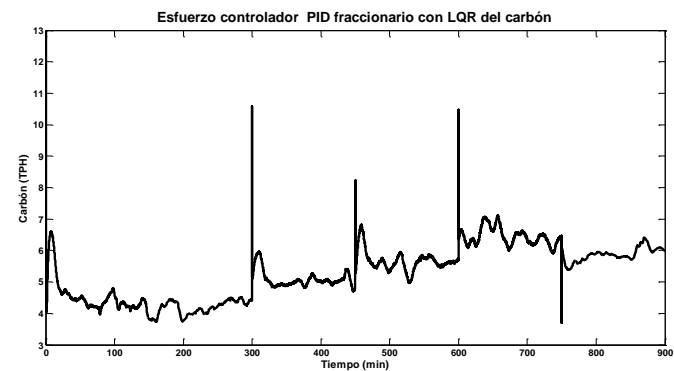

Fig. 5. Esfuerzo de controlador PID fraccionarios con LQR de carbón.

Fuente: Autores

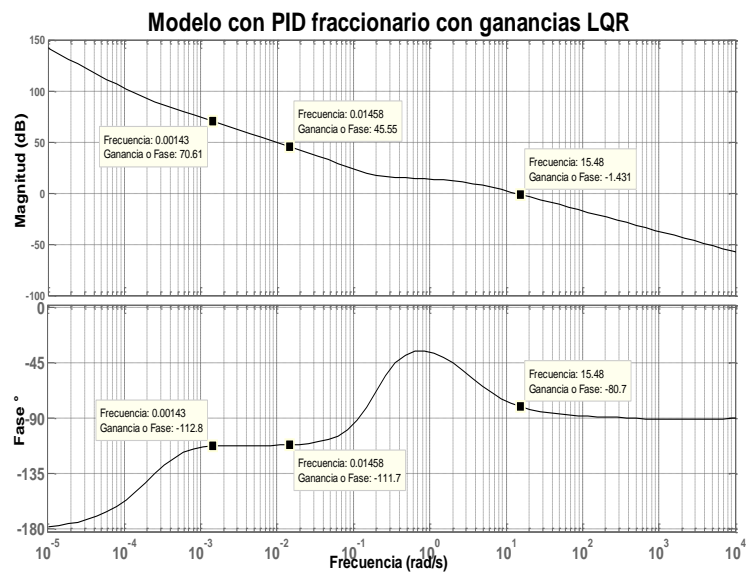

Fig. 6. Diagrama de Bode de modelo PID fraccionario con ganancias corregidas.

Fuente: Autores

El comportamiento del proceso de lazo cerrado en el dominio del tiempo ante variaciones de ganancia se presenta en la Fig. 7. Se observa que los tiempos de estabilización son muy similares y las diferencias en los sobrepasos no son significativas. Este comportamiento verifica la robustez del control ante variaciones de ganancia a la salida del sistema, aunque los rangos de ganancia 
sean inferiores a los del sistema con el control PID fraccionario con las ganancias halladas al tanteo.

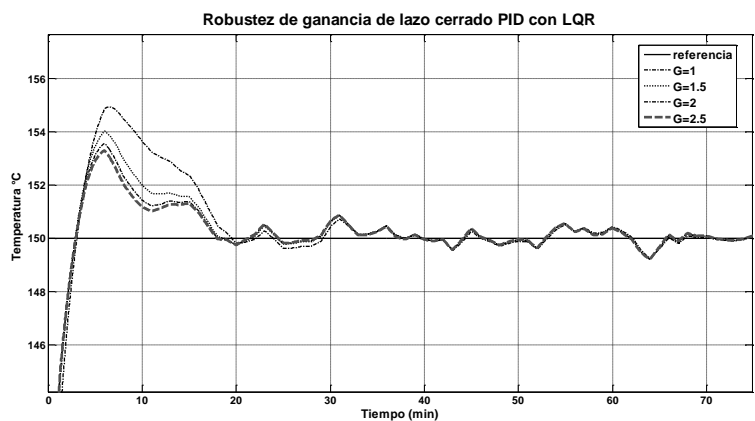

Fig. 7. Robustez de ganancia de C(s)G(s) con PID fraccionario de lazo cerrado. Fuente: Autores

La sensibilidad determina el comportamiento del sistema ante cambios en las señales de salida. En la Fig. 8 se observa que a frecuencias inferiores a $0.02571 \mathrm{rad} / \mathrm{s}$, el sistema tiene ganancias inferiores a $-40 \mathrm{~dB}$. Por último, se evaluó el criterio de rechazo de ruido de alta frecuencia y los resultados se aprecian en la Fig. 9. Se observa que señales con ruido superior a $89.36 \mathrm{rad} / \mathrm{s}$ tendrán una atenuación menores a $-20 \mathrm{~dB}$, esto garantiza que el control es robusto ante perturbaciones de alta frecuencia.

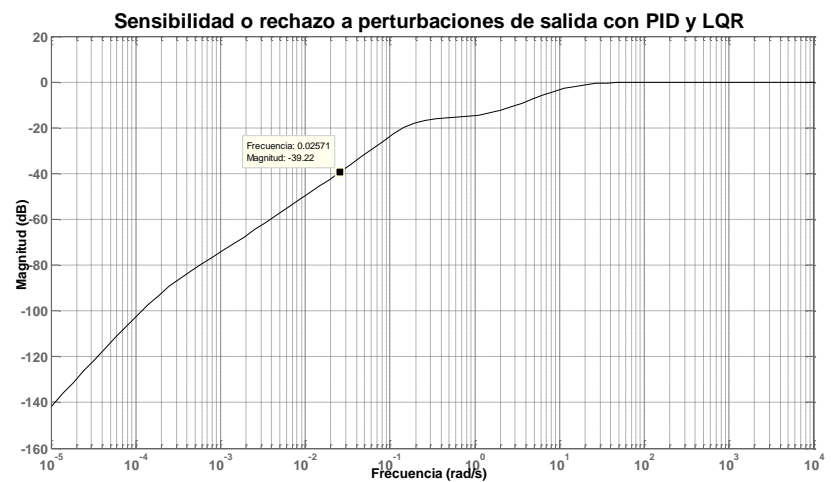

Fig. 8. Sensibilidad o rechazo a perturbaciones de salida con PID y LQR.

Fuente: Autores 


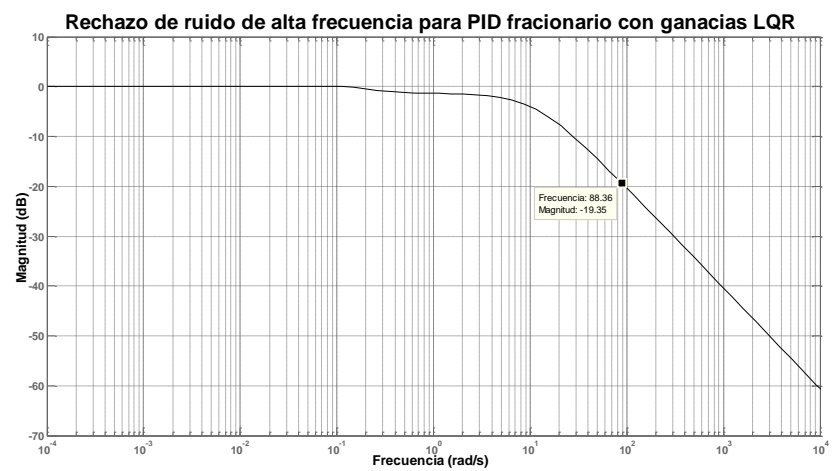

Fig. 9. Rechazo de ruido de alta frecuencia para PID con ganancia LQR. Fuente: Autores

\section{CONCLUSIONES}

El controlador se diseñó utilizando dos criterios de controladores robustos, uno fue el diseño del controlador PID de orden fraccionario y el otro fue el controlador LQR. En el controlador PID se remplazó la ganancia proporcional Kp por la ganancia de la matriz hallada para el LQR, esto permitió controlar la temperatura de los gases de salida del horno actuando sobre tres variables críticas del proceso, tales como la dosificación de carbón, la alimentación de pasta y la dosificación del agua de enfriamiento. Para verificar la validez de la metodología empleada en el diseño del controlador se realizó un análisis de criterios de robustez al sistema controladorplanta, arrojando resultados satisfactorios para el desempeño de los controladores y de la señal de salida en estado transitorio y en estado estable.

Los análisis de sensibilidad y rechazo de ruido a alta frecuencia determinan el rango de frecuencias de trabajo del sistema diseñado. Para el caso de este proceso, el rango es amplio (0.020 a $90 \mathrm{rad} / \mathrm{s}$ ), lo cual contribuye con la robustez del sistema en un rango amplio de frecuencias. 


\section{AGRADECIMIENTOS}

Este trabajo se logró gracias a Cementos Argos S.A. por permitir el uso de bases de datos de proceso de cemento blanco, al Instituto Tecnológico Metropolitano y el Grupo de Investigación Automática y Electrónica por su apoyo en el proyecto PM 12104.

\section{REFERENCIAS}

Feng, G., Bin, L., Xiaochen, H., \& Peng, G. (2010). Research on the fuzzy predictive control for calcining temperature of the rotary cement kiln. IEEE 10th INTERNATIONAL CONFERENCE ON SIGNAL PROCESSING PROCEEDINGS, 2568-2571.

Holmblad, L. ., \& Østergaard, J.-J. (1995). The FLS application of fuzzy logic. Fuzzy Sets and Systems, 70(2-3), 135-146.

Li, Z. (2010). Support Vector Machine Model Based Predictive PID Control System for Cement Rotary Kiln. System, 3117-3121.

Lin, X., Liu, T., Cao, D., \& Huang, Q. (2009). Temperature Control in Cement Rotary Kiln with Neural Network-Based Heuristic Dynamic Programming. Advances in Neural Networks-ISNN 2009, 5552(PART 2), 1078-1086.

Lin, X., Liu, T., Song, S., \& Song, C. (2009). Neuro-controller of cement rotary kiln temperature with adaptive critic designs. In 2009 IEEE Symposium on Adaptive Dynamic Programming and Reinforcement Learning (pp. 1-5). Ieee.

Marquez Martinez, M. (1989). Combustión y Quemadores (Primera., pp. 27-40). Barcelona: Marcombo S.A.

Pani, A. K., Vadlamudi, V. K., \& Mohanta, H. K. (2013). Development and comparison of neural network based soft sensors for online estimation of cement clinker quality. ISA transactions, 52(1), 19-29.

Shahriari, K., \& Tarasiewicz, P. E. S. (2009). A Model-Based Approach for Clinker Rotary Kiln Monitoring and Instrumentation. 2009 IEEE Cement Industry Technical Conference Record, 1-11.

Vinagre, B. M., \& Monje, C. A. (2006). Introducción al Control Fraccionario. Revista Iberoamericana de automatica e Informatica Industrial, 3, 5-23.

Wang, C., Wang, S., Yu, G., \& Li, X. (2010). Application Research of a Fault Diagnosis Expert System for Cement Kiln Based on .Net Platform. 2010 Second International Conference on Intelligent HumanMachine Systems and Cybernetics, 208-212. 
Wang, P., \& Kwok, D. P. (1992). Analysis and synthesis of an intelligent control system based on fuzzy logic and the PID principle. Intelligent Systems Engineering, 1(2), 157.

Wang, S., Dong, F., \& Yuan, D. (2007). The Design and Implementation of a Cement kiln Expert System. 2007 IEEE International Conference on Automation and Logistics, 2716-2719.

Yongjian, W., Minghao, T., Xiaojie, Z., \& Tianyou, C. (2007). The Design and Application of Hybrid Intelligent Control System for Rotary Kiln Process in Alumina Production. 2007 Chinese Control Conference, 222-227. 